\title{
Video Analysis of Side Hop Technique in Trials
}

\author{
Rodolfo Vastola, Silvia Coppola, Albano Daniele, and Francesca D'Elia \\ Motion Analysis Laboratory, Department of Human, Philosophical and Educational Sciences, University of Salerno, Fisciano \\ Salerno 84084, Italy
}

\begin{abstract}
Trials is a specialty of off-road cycling in which the rider has to face obstacle courses without resting feet on the ground. Technique in this sport has a great importance, since it reduces the risk of committing penalties and allows more efficient execution of the gesture. To improve technique, the motion analysis allows to study the gesture both qualitatively and quantitatively. In this work video analysis was used to study the side hop from rear wheel technique. Two different executions of this technique were analyzed. The primary purpose is the identification of the phases that make up the technical gesture. It was given an explanation to the movement strategies adopted in the execution of the jump in the two different situations.
\end{abstract}

Key words: Bike trials, cycling, vertical jump, video analysis, sport technique.

\section{Introduction}

Trials is one of the specialties of the off-road cycling U.C.I. (International Cycling Union) and among them it is one that requires greater control on the bike in very special race situations. The goal is to overcome obstacles grouped into tracks ("zones") without touching the ground with the body or with the bicycle, excluding of course the wheels, to not committing penalties. Having dealt with all areas for a number of laps prescribed, he is declared the winner the driver who committed the least number of penalties [1]. The practice of trial biking originated in the $60 \mathrm{~s}$ in the field of moto trial, when it began to modify normal bicycles to allow more young people try to imitate the motorcycle riders [2]. The most important international events are the World Trials Championship played in a single trial in conjunction with the World Championships of other specialties of the off-road cycling and Trials World Cup which instead provides more races in different locations during the racing season. For young people up to age 16 are held the Trials World Youth Games. Trials bikes must combine strength and lightness, for this reason are used

Corresponding author: Rodolfo Vastola, Ph.D., assistant professor, research fields: sports and motor performance analysis. aeronautical materials for their construction that allow to withstand the stress imposed by this kind of sport while keeping weight to a minimum at around $7 / 8 \mathrm{~kg}$. The most common sizes for trials bicycles are 20", 26", 24 " which refer to the diameter of the wheels, there are also bike less than 20" for the children [3]. The choice of the ideal measure is not determined only by the height of the rider, this is a factor to take into account, but missing the saddle and being able to change the driving position with longer stems this factor doesn't really matter. Generally high tendency towards 24 " and 26 " drivers while lower rider to the 20 ", but the greatest impact on the choice is the origin of the rider, who comes from the mountain bike may prefer the 26" while those who rode BMX will have more feeling with the $20 "$. These guidelines are very general, what really influences the choice of the optimal size are the driving sensation the rider experiences, so only after trying various sizes and settings it can make the right choice according to personal preferences. It is generally agreed that the skills, control and driving experience acquired in trials practice, are essential to develop the required technical skills from other cycling and motorcycling sports. Technique not only plays a role in the improvement of sports performance, the learning and the use of an optimal technique have 
strong repercussions in the field of health for the prevention and reduction of injuries, in fact an economic, efficient, powerful gesture, is based on a better positioning of the body under load, this allows less stress of the structures involved with a risk of injuries decrease [4]. In trials, in accordance with the technical classification of sports, technique could lie in between those disciplines that require an adaptation in variable situations (situational sports) and those in which the technique saves energy to complete the performance (endurance sports) [5]. In trials riding, the rider has to complete an obstacle course in a time of 2 minutes, trying to limit errors (penalty) under conditions which vary from one location to another, both for the obstacles (layout, shape, material), both for the environmental conditions (rain, wind) and terrain (slippery, compact), which require significantly adaptations in the race, it adds to this the fact that in the execution of movements large muscle groups are involved and also an external load (the bicycle) must be moved several times during the race. By this, we understand the technical predominant role in limiting the energy consumption maintaining a state of "freshness". Technical skills in this sport are generally classified in two types, to go up or down the obstacles. The side hop is used in the practice of trials to overcome obstacles in situations where you cannot use a running start. Of this jump are implementing variants that differ from one another both in the initial phase and the final. With regard to the first part, the jump may be performed starting with both wheels in contact with the ground or from the balance on the rear wheel. In the final part, we might call the approach to the obstacle, the various methods differ mainly in the contact sequence of the wheels on the obstacle, in this case there are three types of jump finalization. One type provides a complete overcoming of the obstacle with both wheels, a wheel in the other two types precedes the other. In the case of initial contact with the front wheel is seeing a forward displacement of the body and a locking of the front wheel to be used as a pivot to assist the rise of the rear wheel. The finalization of the jump varies according to the type of obstacle which is required to deal with, whether or not this offers the possibility to exploit, for example, the "front wheel pivot action" or on the contrary does not allow any contact, but obliges to cross completely the obstacle.

About sport technique improvement, video analysis is one of the most useful tools. In terms of feedback, video footage is the most objective source, because the video provides the most realistic form of feedback and understanding [6]. Thanks to increasingly lower costs of modern cameras, video capture has become the means most used for performance analysis at any level, as it allows to obtain qualitative and quantitative data is compared to biomechanical analysis, which type notational [7]. Video footage are executed through the use of digital cameras while the processing work is entrusted to specific software, some available for free as Kinovea offering basic functions, other for professional purpose. Elaboration post-acquisition can be obtained information about location, trajectories, joint angles, speed and acceleration. The most important characteristics of a camera for this type of operations are the image resolution and speed of acquisition in fps (frames per second), in these investigations postural occupy a marginal role, as opposed to analysis of fast and complex gestures are necessary high-performance instrument.

In this work, two execution methods on two different obstacles were analyzed. A jump has been performed on an obstacle of cubic form and was performed using the front wheel pivot finalization mode. The other jump was performed on a bar placed on two supports, given the nature of the obstacle the jump was performed overcoming the bar. Both jumps were performed from the rear wheel balance position.

\subsection{State of Research on Trials}

From the research carried out, it is present in the literature a single work inherent in the trials, concerning the possible developments in the study of 
this sport through a dedicated bicycle equipped with a power meter applied to the transmission [8] and a work on use of optoelctronic systems for the analysis of technique in trials [9]. This scarcity of studies demonstrates how this discipline is still young and little studied. On the other hand, there are numerous works on cycling biomechanics particularly regarding the most practiced specialties related aspects such as cycling, positioning on the bike, muscle activation, the mechanisms involved energy, aerodynamics, however, being trials very different from these specialties, data collected from these jobs are of little use [10]. Another exception rather deserves the studies on the BMX (bicycle motocross) that presenting the most similarities with the trials, such as driving in a stand up position $[11,12]$.

\subsection{Objectives}

Given the present state of research, this work has the primary purpose of introducing the motion analysis in this discipline. So far, the coaches have been based assessments on personal experience and direct observation of the gesture without any objective feedback as it can be the video. Thanks to the video analysis of the side hop technique, the executions of the jump performed on two different types of obstacle were analyzed. The phases that make up the gesture were identified, calling them carefully delineating the start and end, and giving an explanation to the movement strategies adopted in the two different situations considered.

\section{Methodology}

\subsection{Subject}

The subject participant in this study was a 27 years old male, $1.82 \mathrm{~m}$ height and $77 \mathrm{~kg}$ of body weight, with 8 years of experience in trials riding. Right dominant foot. In trials, the dominant foot refers to the foot which is kept forward when riding the bike.

\subsection{Instruments}

For this work, it was used a camera (Toshiba Camileo SX500, 1280x720, 60fps) for shooting. It was used a tripod to support the camera, adjustable in height and rotation on transverse and sagittal plane.

The bicycle used was an Echo MkII 26", geometry $1080 /+55 / 380 / 72$, with mechanical disc brake on the front and hydraulic rim brake on the rear.

The obstacles were a $1 \times 1 \times 1 \mathrm{~m}$ cube with metal frame and the upper of wood and a bar mounted on two height adjustable metal uprights.

\subsection{Procedure}

Obstacles have been prepared on a flat floor without slopes making sure that the cube and the bar supports were stable.

The camera was positioned orthogonally to the sagittal plane of the action to be analyzed at a height of $1 \mathrm{~m}$ from the ground with inclination angles equal to $0^{\circ}$.

The rear tire was inflated at 1.7 bar. The pressure was monitored before each set of jumps.

Two sets of 3 jumps were performed, a set for type of obstacle. It was taken a rest period between the jumps and between the sets. The rest time between the jumps was 2' and 5 ' between the sets.

A warm up was performed before the jumps. In the beginning, joint mobility exercises were used consisting of large and circular movements of all the joints of the upper and lower limbs, also at the level of the spine. 5' of running at warm up pace were performed and subsequently oscillations of lower and upper limbs. Subsequently a set of 5 squat jumps were performed to approach the dynamics of the gesture [13]. In the end, more specific moves on the bicycle were performed, jumping over the bar from a height of $60 \mathrm{~cm}$ up to $1 \mathrm{~m}$, increasing $10 \mathrm{~cm}$ after each jump.

\subsection{Execution of Trials}

The jumps were performed with the obstacle placed to the right of the subject. Each jump was acquired from preparation to jump at the time of stabilization 
after landing. All jumps were performed from the rear wheel balance position.

\subsection{Data Processing}

The video footages were processed by the video analysis software Kinovea (kinovea.org). This free software allows to take measurements of the kinematic parameters such as joint angles, displacement, velocity and acceleration. Some video editing functions allow to export frames from the video, compare two movies together, draw lines or write on the screen.

This work has proceeded viewing the video frame by frame, in this way it was possible to clearly identify the most interesting moments of each phase. To identify the jump phases, jumps in other disciplines such as the high jump and squat jumps was taken as a reference. Start and stop moments of each phase and the sub-phases within these have been identified. The comparison with other types of jump made possible in addition the identification of the objectives of each phase within the jump. They were later exported the frames of the most significant moments for the gesture description.

\section{Results and Discussion}

By using video analysis, it was possible to identify the following phases of the jump (Fig. 1): (1) Preparation; (2) Loading; (3) Take-off; (4) Flight; (5) Landing.

In the preparation phase, the athlete search for the optimal positioning with respect to the obstacle. In this type of jump, you stand sideways to the obstacle and depending on how you intend to take and the personal style assumes a parallel position or with the front wheel slightly turned towards the obstacle. This phase starts with a stable two-wheel position and ends with the positioning on the rear wheel by seeking stability in this position.

In the loading phase, as well as by one squat jump, you need to accumulate elastic energy in the muscle-tendon structures and release it at the take-off. The compression of the tire also plays a role during this phase. The athlete performs a rapid bending of the lower limbs by searching the compression of the rear
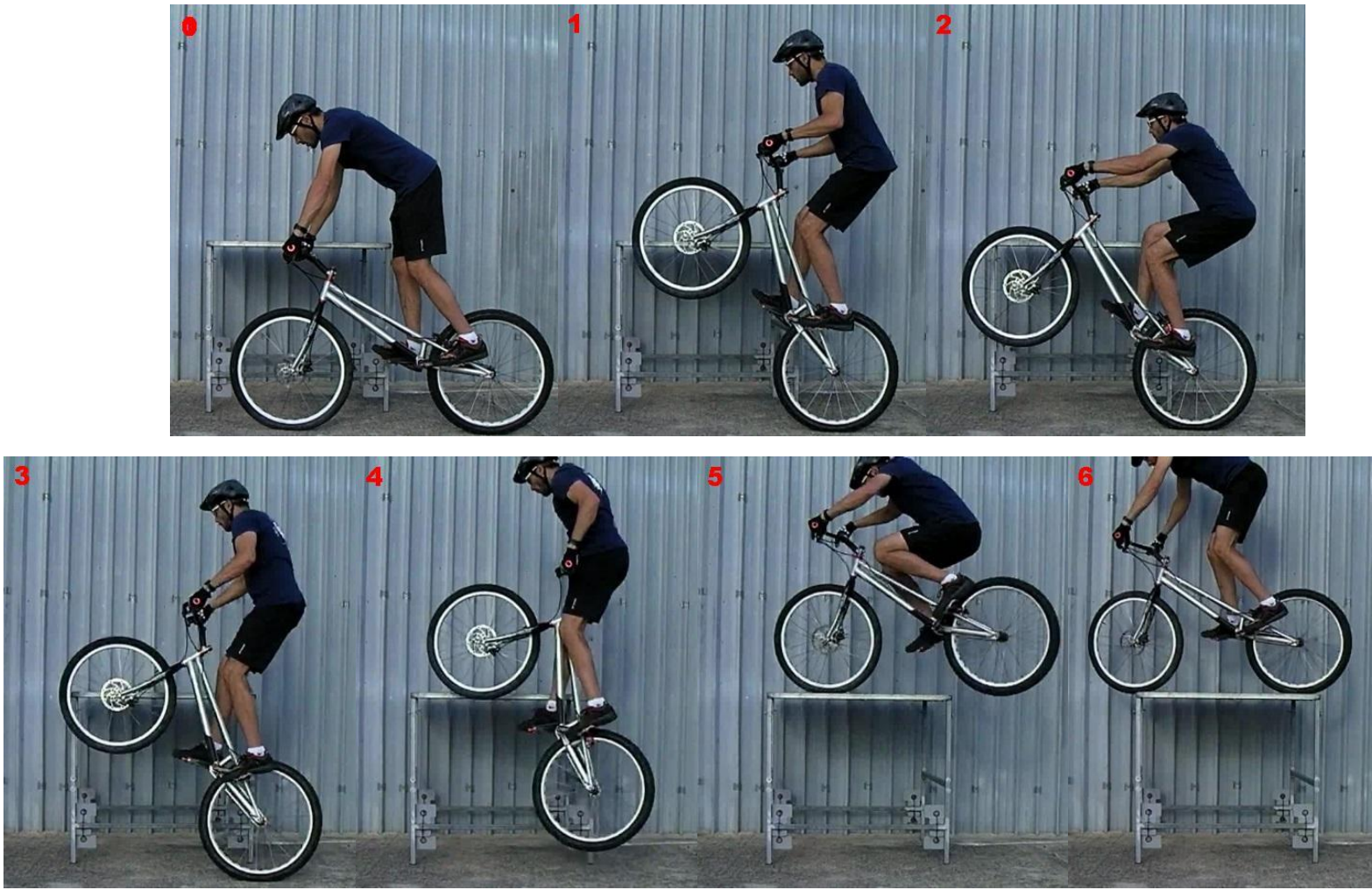
Fig. 1 Side hop: preparation phase $(0,1)$; loading phase (2); take-off phase (3); flight phase (4); landing phase (5, 6).

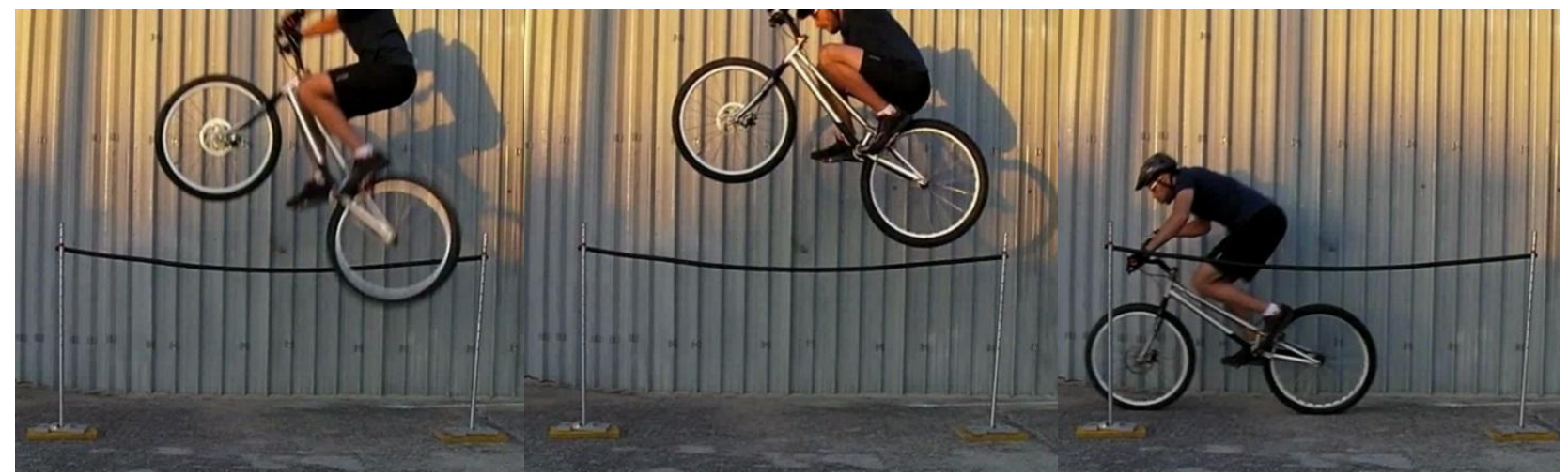

Fig. 2 Side hop performed on the bar. The bar is completely crossed, as shown on the central picture.

tire and the accumulation of elastic energy in the muscle. This phase runs from the beginning of the athlete's descent and ends with the lowest point reached by the hips.

The take-off allows you to release the energy stored in the muscles and the rear tire. At this stage, it comes into play the strength expressed through the bicycle transmission, which develops through a pedal stroke at the take-off. The athlete performs a fast ascent, seeking the full extension of the body upwards, ending with a little pedal stroke prior to the take-off. This phase runs from the beginning of the hips rise at the time of take-off.

The flight phase is characterized by the complete absence of contact points with the ground or the obstacle. In this phase, you reach the maximum extension of the lower limbs immediately after the take-off. In the final stage instead a pull-up action of the lower limbs aims to overcome the obstacle. The goal of this phase is the achievement of the maximum height possible of the athlete-bicycle complex. The start coincides with the take-off and the end can be identified with the leap over the obstacle.

The landing represents the final phase of the jump, based on the technical variant used may coincide with the contact on the obstacle, or with the contact of the wheels to the ground, as in the bar jump (Fig. 2). In this phase, we must handle the contact with the obstacle while maintaining the balance and finding the stability.
The athlete performs damping action by bending the lower limbs, in this way the impact due to landing is absorbed and a greater stability is achieved while keeping the center of mass low. The action ends with the stabilization on two wheels as in the preparation phase.

\section{Conclusions}

This work is a starting point for the motion analysis applied to the sport of trials. The procedure described in this work for video analysis could be a reference for the study of other trials techniques. The name of the phases could provide the basis for a universal terminology to be used in this sport. The data obtained and the methods used could help the coaches for the technical education and improvement of performance in trials. Knowledge of the phases and dynamics that are at the base allow to understand where and why to intervene to correct errors in the execution. The presence of a counter-movement of the lower limbs during the loading phase, could provide guidance for structuring more specific training programs. In the future will be analyzed further aspects of this sport with a greater emphasis on the kinematics of the gesture.

\section{References}

[1] U. C. I. 2015. International Cycling Union. Regulations, Part VII: Trials. Aigle: U. C. I. Accessed August 10, 2016. http://www.uci.ch/mm/Document/News/Rulesandregulati 
on/16/91/28/7-TriaEversionon180315_English.pdf

[2] Happich, J. 2012. Your Complete Guide to Trials Riding. Parigi: Wild Grip Publishing, 164-77.

[3] Limatore, A. Techniques and Tricks for Biking and BikeTrial. Verona: Edicicloeditore, 49-55. (in Italian)

[4] Weineck, J. 2009. The Optimal Training. Perugia: Calzetti \& Mariucci, 149-60. (in Italian)

[5] Scotton, C. 2003. Technical Classification of Sports Specialties. Perugia: Calzetti \& Mariucci, 47-59. (in Italian)

[6] Garhammer, J., and Newton, H. 2013. "Applied Video Analysis for Coaches: Weightlifting Examples." International journal of Sports Science \& Coaching 8 (3): 581-94.

[7] Huges M., Lipoma M., and Sibilio, M. 2010. Performance Analysis. Franco Angeli, 43.

[8] Bertucci, W., and Rogier, S. 2015. "Interest of a New Instrumented Mountain Bike Trial." Annals of Sports Medicine and Research 2 (5): 1030-40.
[9] Vastola, R., Medved, V., Albano, D., Coppola, S., and Sibilio, M. 2016. "Use of Optoelctronic Systems for the Analysis of Technique in Trials." Journal of Sports Science 4 (5): 293-9.

[10] Timmer, C. A. 1991. "Cycling Biomechanics: A Literature Review." Journal of Orthopaedic \& Sports Physical Therapy 14 (33): 106-13.

[11] Gianikellis, K., Skiadopoulos, A., and Bote, A. 2011. “3D Kinematics Applied to the Study of Individual BMX Gate Start Technique." Portuguese Journal of Sport Sciences 11 (2): 30-45.

[12] Redfield R. C., Self, B., Fredrickson, B., and Kinard, A. 2004. "Motion Measurements in the Jumping of a Mountain Bike." Journal of Instrumentation, Systems and Automation Society 40: 43-50.

[13] Bobbert, M. F., Gerritsen, K. G., Litjens, M. C., and Van Soest, A. J. 1996. "Why Is Countermovement Jump Height Greater than Squat Jump Height?" Medicine and Science in Sports and Exercise 28 (11): 1402-12. 